\title{
Resistance Exercise Performed at Different Degrees of Arterial Occlusion Pressure does not Induce Prolonged Oxidative Stress or Muscle Damage
}

\section{Eduardo DS Freitas ${ }^{1,2 *}$, Michael G Bemben ${ }^{1}$, Alexandre S Silva ${ }^{3}$, Rodrigo R Aniceto ${ }^{2,4}$, João B Ferreira- Junior ${ }^{5}$ and Maria S Cirilo-Sousa ${ }^{2}$}

${ }^{1}$ Department of Health and Exercise Science, Neuromuscular Research Laboratory, University of Oklahoma, USA

${ }^{2}$ Department of Physical Education, Kinanthropometry and Human Performance Laboratory, Federal University of Paraiba, Brazil

${ }^{3}$ Department of Physical Education, Laboratory of Studies in Physical Training Applied to Performance and Health, Universidade Federal da Paraiba, Brazil

${ }^{4}$ Department of Physical Education, Study and Research Group in Weight Training and Psychophysiological Responses, Integrated Colleges of Patos, Brazil

${ }^{5}$ Federal Institute of Southeast of Minas Gerais - Campus Rio Pomba, Brazil

*Corresponding author: Eduardo DS Freitas, Department of Health and Exercise Science, Neuromuscular Research Laboratory, University of Oklahoma, 1401 Asp Ave, HHC 104 - Room 06, Norman, OK 73019, USA, E-mail: eduardofreitas@ou.edu

\begin{abstract}
This study investigated if resistance exercise performed at differing Arterial Occlusion Pressures (AOP) causes oxidative stress and muscle damage. Twelve males completed 4 sets of 10 repetitions of knee extension at $20 \%$ of $1 \mathrm{RM}$, with $30 \mathrm{~s}$ rest intervals between sets, that varied only in the amount of restriction pressure applied: 1) CON (no pressure), 2) AOP-50 (50\% of the total AOP), 3) AOP-75 (75\% of the AOP), and 4) AOP-100 (100\% of the AOP), with each condition separated by 7 days. Ratings of Delayed-Onset Muscle Soreness (DOMS), Maximal Isometric Strength (MIS), serum levels of Creatine Kinase (CK), Lactate Dehydrogenase (LDH), Lipid Peroxide (LP), and the Antioxidant Activity of Plasma (AAP) were assessed at rest, 1, 24, and $48 \mathrm{~h}$ post-exercise. DOMS, $\mathrm{MSI}, \mathrm{CK}, \mathrm{LP}$, and AAP did not differ among the conditions $(p>$ 0.05 ) across the different time points, however, plasma levels of LDH were significantly lower $(p<0.01)$ at $24 \mathrm{~h}$ post-exercise $(265.83 \pm 17.55 \mathrm{UI} / \mathrm{L})$ in comparison to $48 \mathrm{~h}$ post exercise (294.96 $\pm 17.51 \mathrm{UI} / \mathrm{L})$ averaged across all groups. It was concluded that resistance exercise with differing occlusion pressures does not cause prolonged muscle damage or oxidative stress, regardless of the amount of AOP.
\end{abstract}

\section{Keywords}

Blood flow restriction, KAATSU, Strength exercise, Metabolic stress, Occlusion training

\section{Introduction}

Exercise-induced muscle damage has been shown to occur following acute bouts of high-intensity resistance exercise, mostly due to the eccentric component of the contractions performed [1-3]. Although some researchers have investigated the physiological mechanisms underlying the muscle damage phenomenon in both human and animal models, these responses remain unclear. Several factors contribute to muscle damage following resistance exercise, such as gender [1], exercise volume [4], rest periods between sets [5], and the type of the exercise performed [1].

Concerning exercise intensity, there is no evidence that low-intensity resistance exercise is capable of inducing significant muscle damage post-exercise [6]. However, low-intensity resistance exercise (20-50\% 1RM) with Blood Flow Restriction (BFR) has gained some attention regarding the occurrence of muscle damage linked to this model of exercise [7] and it is speculated that it may cause minimal muscle damage $[8,9]$.

Oxidative stress has been linked to muscle damage

Citation: Freitas EDS, Bemben MG, Silva AS, Aniceto RR, Ferreira-Junior JB, et al. (2017) Resistance Exercise Performed at Different Degrees of Arterial Occlusion Pressure does not Induce Prolonged Oxidative Stress or Muscle Damage. Int J Sports Exerc Med 3:075. doi.org/10.23937/2469-5718/1510075 Received: April 26, 2017; Accepted: September 19, 2017; Published: September 21, 2017 Copyright: (C) 2017 Freitas EDS, et al. This is an open-access article distributed under the terms of the Creative Commons Attribution License, which permits unrestricted use, distribution, and reproduction in any medium, provided the original author and source are credited. 
following resistance exercise [10]. In this sense, muscle damage related to BFR could be better explained by internal physiological factors rather than exercise workloads, since BFR exercise uses significantly lower loads (20\%-50\% $1 \mathrm{RM})$ compared to traditional resistance training (70\%$80 \%$ 1RM). Thus, muscle damage observed following BFR exercise may be triggered by the formation of hypoxia-induced oxygen species in the active muscles. During BFR resistance exercise, pneumatic cuffs are attached to the exercising limbs, inflated to a target pressure, and then deflated following completion of the exercise. The venous pooling of blood in the limbs and the resultant increased venous return from the muscle following cuff deflation promotes the creation of reactive oxygen species which could potentially damage muscle tissue [11]. However, it is still unclear whether low-intensity resistance exercise at distinct restrictive pressures $(50 \%, 70 \%$, or $100 \%$ of total occlusion) would be able to induce significant acute oxidative stress and muscle damage.

The majority of studies that have attempted to examine the muscle damage response after BFR exercise have a common limitation that needs to be addressed. Most of these studies have applied an arbitrary pressure $[9,12]$ or have based the restrictive pressures on the subjects' brachial Systolic Blood Pressure (SBP) $[8,13]$. By applying the same amount of pressure to all subjects, it is very likely that subjects with different size limbs would experience differing amounts of BFR resulting in either an overestimation or underestimation of the blood flow going to the working muscle. Additionally, studies that have used SBP at the brachial artery (arm) to determine the restrictive pressure for the lower limbs may underestimate the restrictive effects of the pressured being used. Recent evidence suggests that the Arterial Occlusion Pressure (AOP) should be individually prescribed for each subject $[14,15]$ and based on the amount of pressure required to totally occlude blood flow to the limbs [16]. Since occlusion pressure, as an independent factor, plays an important role in the muscle damage response to BFR resistance exercise due to the ischemia-reperfusion phenomena, different degrees of BFR should be evaluated.

To the best of our knowledge, no study has examined how oxidative stress and muscle damage responses are affected by BFR resistance exercise performed at different degrees of occlusion pressure. The fact that a wide range of occlusion pressures could be applied during BFR resistance exercise and that muscle performance is reduced by muscle damage, new information in this area should be helpful for physiotherapists, athletic trainers, and strength and conditioning trainers to improve BFR exercise training programs. Therefore, the purpose of the current study was to examine if BFR resistance exercises performed at different degrees of AOP cause oxidative stress and muscle damage in young trained men. Taking into consideration that muscle damage after BFR exercise can be triggered by the hypoxia-induced oxygen species formation in the ac- tive muscles [11], we hypothesized that resistance exercise with a higher AOP would result in a higher oxidative stress response and greater muscle damage, as well as a longer recovery period.

\section{Methods}

\section{Subjects}

Twelve trained males $(20.58 \pm 2.39 \mathrm{yrs}, 72.77 \pm 8.42 \mathrm{~kg}$, $1.79 \pm 0.07 \mathrm{~m}, 22.71 \pm 1.71 \mathrm{~kg} / \mathrm{m}^{2}$ ) involved in resistance training exercise for at least 6 months prior to this study were included. Only healthy, non-smoking subjects (without any history of cardiovascular or osteomuscular disease, non-obese, with BMI values between 20.0 and 29.9 $\mathrm{kg} / \mathrm{m}^{2}$, with an ankle-brachial index between 0.9 and 1.30 , and without any known injuries) were included. Subjects were asked to avoid any resistance exercise throughout the study period and to refrain from alcohol and caffeine at least $24 \mathrm{~h}$ prior to each exercise session. The study protocols and procedures were explained to each subject, and written informed consent was obtained prior to participation. This study was approved by the Institutional Review Board of the Federal University of Paraiba.

\section{Experimental design}

Subjects were required to complete all four experimental conditions, which varied according to the amount of AOP applied to the lower-body. The four experimental conditions were performed using a contralateral counterbalanced design as follows: 1) Unilateral knee extension with no AOP (CON); 2) Unilateral knee extension at $50 \%$ of AOP (AOP-50) using the contralateral leg; 3) Unilateral knee extension at $75 \%$ of AOP (AOP-75); and 4) Unilateral knee extension at $100 \%$ of AOP (AOP-100) using the contra lateral leg. Each experimental condition was separated by 7 days. The percentages of blood flow restriction ( $0 \%$, $50 \%, 75 \%$, and $100 \%$ ) were based on the pressure required to totally occlude the arterial blood flow to the lower extremities. The order of the experimental conditions were randomized by using a random numbers table.

Participants attended the laboratory on several different occasions. On the first visit, height, weight, resting arterial blood pressure, $A B I$, and AOP were measured. Subjects also completed a standardized maximal strength test (one Repetition Maximum-1RM) for both knee extensors (right and left legs), such that $20 \%$ of 1 RM could be determined for each experimental condition. In subsequent visits, subjects performed the experimental conditions, and the oxidative stress and muscle damage markers were measured before and at $1 \mathrm{~h}, 24 \mathrm{~h}$, and $48 \mathrm{~h}$ following each exercise protocol.

\section{Determination of the Arterial Occlusion Pressure}

The pressure required to achieve total AOP for each leg was determined using a standardized protocol first proposed by Laurentino, et al. [16]. With subjects in a supine position, a vascular Doppler probe (Medpej, DV-2001, Bra- 
zil) was placed on the tibial artery to detect the auscultator pulse. Then, a standard $15 \mathrm{~cm}$ wide blood pressure cuff [17] located at the most proximal portion of the thigh was inflated until the pulse could no longer be detected and then slowly released until the moment in which the pulse could be detected again. This value was considered to be $100 \%$ of the AOP and was used to determine the different percentages of occlusion pressure used in the different exercise sessions $(0 \%, 50 \%, 75 \%$, and $100 \%)$.

\section{RM test}

The 1RM test was randomly performed for each leg separately as described by Baechle and Earle [18]. The initial warm-up period utilized a weight that could be easily lifted for 5-10 repetitions of knee extension. Then, following a 1 min rest period, the weight was increased by $10-20 \%$, and 5 more repetitions were performed. The last warm-up set of 2-3 repetitions occurred after a 2 min rest, with another weight increase of $10-20 \%$. Participants then began single attempts of knee extension with increased weight until the weight could not be lifted through a full range of motion. No more than 5 attempts were allowed to reach maximal strength, and $3 \mathrm{~min}$ rest periods separated each attempt. After a $10 \mathrm{~min}$ rest, the second leg performed the same test sequence. There was no significant difference between the maximum strength values measured from both legs $(p>0.05)$.

\section{Oxidative stress}

Oxidative stress was verified according to the Lipid Peroxide (LP) levels and the Antioxidant Activity of Plasma (AAP). The lipid peroxide levels were quantified using the reaction of thiobarbituric acid with the decomposition products of the hydroperoxides, as described by Ohkawa, et al. [19]. For this, $250 \mu \mathrm{L}$ of serum was incubated in a water bath at $37{ }^{\circ} \mathrm{C}$ for 60 minutes. Samples were then precipitated with perchloric acid $\mathrm{AA}$ at $35 \%$ and centrifuged at $14,000 \mathrm{rpm}$ for 20 minutes at a temperature of $4^{\circ} \mathrm{C}$. The top layer of supernatant was transferred to new micro tubes, to which $400 \mu \mathrm{L}$ of thiobarbituric acid at $0.6 \%$ was added and incubated at $100{ }^{\circ} \mathrm{C}$ for 60 minutes. After cooling, the samples were analyzed using an ultraviolet spectrophotometer (Bioespectro, model 22, Brazil) at a wavelength of $532 \mathrm{~nm}$ and room temperature.

The antioxidant activity of the plasma was determined in agreement with Brand-Williams, et al. [20]. In detail, an aliquot of $1.25 \mathrm{mg}$ of 2,2-Diphenyl-1 Picrylhydrazyl (DPPH•) was diluted in $100 \mathrm{ml}$ of ethanol $(99.5 \%$ absolute ethanol), kept under refrigeration, and protected from the light (using aluminum foil or amber-colored glass). In appropriate centrifuge tubes, $3.9 \mathrm{ml}$ of $\mathrm{DPPH} \bullet$ solution was added, followed by $100 \mu \mathrm{L}$ of plasma. The tubes were vortexed and left to stand for 30 minutes. Then, tubes were centrifuged for 15 minutes at a speed of $10,000 \mathrm{rpm}$ and a temperature of $20^{\circ} \mathrm{C}$. The supernatant was used to perform the analysis in a spectrophotometer at $515 \mathrm{~nm}$. The results are expressed as percentages of the antioxidant activity, using the following equation:

$$
A A P=100-[D P P H \bullet R] t /[D P P H \bullet R] B ~ 100)
$$

$[D P P H \bullet R]$ t and $[D P P H \bullet R] B$ correspond to the remaining concentration of DPPH• after 30 minutes, assessed on the sample ( $\mathrm{t}$ ) and on the white (B) and prepared with distilled water.

\section{Muscle strength}

The maximal isometric strength of the exercising leg was measured using a portable digital dynamometer (Instrutherm Ltd., DD-300, Brazil) attached to the knee extension machine. Participants had their knee positioned at an angle of $60^{\circ}$ (where $0^{\circ}$ represented a full knee extension), and a belt was fastened across the hip to minimize extraneous body movements, which could affect the strength measurements. Subjects were then asked to perform three maximum voluntary contractions and to hold each for 3 seconds. One minute of rest was given between each attempt. The highest score was registered and used for statistical analysis. In addition, a familiarization session was performed for the first week, before the main testing.

\section{Blood markers of muscle damage}

Serum levels of Creatine Kinase (CK) and Lactate Dehydrogenase (LDH) were used as indirect biomarkers of muscle damage. Venous blood samples $(5 \mathrm{ml}$ for each point of measurement) were obtained from the antecubital vein by a nurse; the samples were placed in assay tubes and centrifuged at a speed of 3,000 rpm for $15 \mathrm{~min}$. The serum was stored at $-20{ }^{\circ} \mathrm{C}$ until analysis. An immunochemical automatic analyzer (Labtest, Labmax 240 premium, Brazil) was used to run the analysis using specific kits for CK and LDH (Labtest, Brazil). All the procedures were performed according to the manufacturer's specifications.

\section{Delayed-onset muscle soreness}

Delayed-Onset Muscle Soreness (DOMS) was measured through an analogic verbal scale proposed by Nieman, et al. [21]. This scale ranges from 0 to 10 as follows: 0 (no soreness), 2 (dull, vague ache), 4 (slight soreness), 5 (more than slight soreness), 7 (sore), 8 (very sore), and 10 (unbearably sore). Subjects were introduced to the scale and asked if they had any questions.

\section{Exercise protocol and blood flow restriction}

During each exercise session, subjects performed 4 sets of 10 repetitions of unilateral knee extensions, through a $90^{\circ}$ range of motion, with a load of $20 \%$ of $1 \mathrm{RM}$ and a pace of 1.5 seconds for both the concentric and the eccentric portion of the contraction. A total of 30 seconds of rest were allowed between sets. BFR was used in all conditions, except for the CON, varying only in the amount of pressure applied. In this regard, the cuff was positioned on the inguinal portion of the exercising limb and then inflated to the target pressure just 
Table 1: Comparison of lipid peroxide levels (nmol/L) at rest, $1 \mathrm{~h}, 24 \mathrm{~h}$, and $48 \mathrm{~h}$ post-resistance exercise performed at different levels of AOP $(n=12)$.

\begin{tabular}{|c|c|c|c|c|}
\hline \multirow[b]{2}{*}{ Time point } & \multicolumn{4}{|c|}{ AOP pressures } \\
\hline & $\operatorname{CON}(0 \%)$ & AOP-50 & AOP-75 & AOP-100 \\
\hline Rest & $2.74 \pm 0.12$ & $3.02 \pm 0.17$ & $2.76 \pm 0.10$ & $2.85 \pm 0.17$ \\
\hline $1 \mathrm{~h}$ post & $2.94 \pm 0.20$ & $2.85 \pm 0.17$ & $2.84 \pm 0.16$ & $2.84 \pm 0.21$ \\
\hline 24 h post & $2.85 \pm 0.11$ & $2.81 \pm 0.12$ & $2.89 \pm 0.18$ & $2.77 \pm 0.21$ \\
\hline 48 h post & $2.96 \pm 0.09$ & $3.05 \pm 0.22$ & $3.07 \pm 0.15$ & $2.85 \pm 0.17$ \\
\hline
\end{tabular}

CON: Control condition with no occlusion; AOP-50: Experimental condition at 50\% of Arterial Occlusion Pressure; AOP-75: Experimental condition at $75 \%$ of Arterial Occlusion Pressure; AOP-100: Experimental condition at $100 \%$ of Arterial Occlusion Pressure.

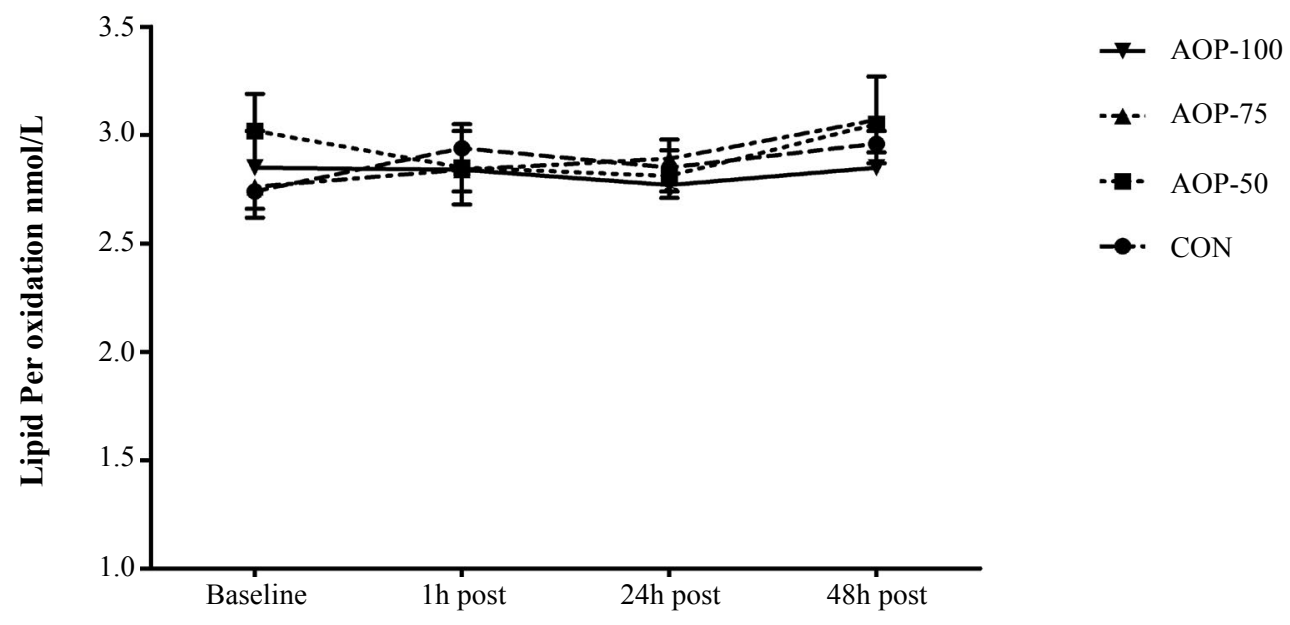

Time points

Figure 1: Comparison of lipid peroxide levels (nmol/L) at rest, $1 \mathrm{~h}, 24 \mathrm{~h}$, and $48 \mathrm{~h}$ after resistance exercise performed at different levels of AOP $(n=12)$. CON: Control condition with no occlusion; AOP-50: Experimental condition at $50 \%$ of Arterial Occlusion Pressure; AOP-75: Experimental condition at 75\% of Arterial Occlusion Pressure; AOP-100: Experimental condition at $100 \%$ of Arterial Occlusion Pressure.

prior to the start of the first set; it was deflated at the end of the fourth set. The amount of AOP for each condition was $66.58 \pm 9.72 \mathrm{mmHg}$ (AOP-50), $99.25 \pm 14.95$ $\mathrm{mmHg}$ (AOP-75), and $129.50 \pm 18.73$ (AOP-100). There was a significant difference between the conditions.

\section{Statistical analyses}

Sample size was calculated using $G^{*}$ Power version 3.1.9.2 for OS X (Franz Faul, Universitat Kiel, Germany) and was set at 12 subjects, corresponding to an effect size of 0.60 , a power of $0.80, \alpha=0.05$, test family $=F$ test and statistical test = ANOVA repeated measures, within-between interactions [22]. The Shapiro-Wilk test was performed to confirm the data normality. A two-way repeated measures ANOVA (condition [CON, 50-AOP, 75-AOP, and 100-AOP] $x$ time [rest, $1 \mathrm{~h}, 24 \mathrm{~h}$, and $48 \mathrm{~h}$ post-exercise]) was used to compare the main effects of time, condition, and the condition by time interaction. When a significant difference was found, a Bonferroni post hoc test was carried out to identify where the difference was located. Given that the delayed-onset muscle soreness data did not present a normal distribution, the Kruskal-Wallis test was used with the Mann Whitney $\mathrm{U}$ test to find mean differences according to Bonferroni corrections. Data are presented as the mean \pm SE. The significance level was set as $p<0.05$. Data were ana- lyzed using SPSS version 22 for OS X (IBM, Chicago, IL).

\section{Results}

\section{Oxidative stress}

As presented in Table 1 and Figure 1 , no significant condition ( $F=0.16, p=0.92)$ or time $(F=0.70, p=0.56)$ main effects and no significant condition by time interaction ( $F=0.56, p=0.83$ ) were observed for LP. For the AAP, there was no significant time main effect $(F=1.55$, $p=0.22$ ) or significant condition by time interaction ( $F$ $=1.55, p=0.18)$, but there was a significant condition main effect $(F=3.32, p=0.03)$; however, follow up analyses reviewed that such difference does not exist $(p>$ 0.05) (Table 2 and Figure 2).

\section{Muscle damage}

As presented in Figure 3, there was no significant condition main effect $(F=1.00, p=0.40)$ or condition by time interaction ( $F=1.21, p=0.30$ ) for changes in the levels of maximal isometric strength, but there was a significant time main effect $(F=3.30, p=0.04)$; however, follow up analysis revealed that only the comparison between the time points at $1 \mathrm{~h}$ post-exercise (79.95 \pm $3.79 \mathrm{UI} / \mathrm{L})$ and at $24 \mathrm{~h}$ post-exercise $(84.27 \pm 3.70 \mathrm{UI} / \mathrm{L})$ approached statistical significance $(p=0.08)$. 
Table 2: Comparison of the antioxidant activity of plasma (\%) at rest, $1 \mathrm{~h}, 24 \mathrm{~h}$, and $48 \mathrm{~h}$ post-resistance exercise performed at different levels of AOP $(n=12)$.

\begin{tabular}{|l|l|l|l|l|}
\hline \multicolumn{3}{l}{ AOP pressures } & & \\
\hline Time point & CON (0\%) & AOP-50 & AOP-75 & AOP-100 \\
\hline Rest & $35.76 \pm 3.36$ & $35.76 \pm 3.33$ & $44.85 \pm 4.25$ & $44.88 \pm 5.39$ \\
\hline 1 h post & $43.70 \pm 2.41$ & $34.76 \pm 3.86$ & $37.15 \pm 3.78$ & $41.76 \pm 4.93$ \\
\hline 24 h post & $39.36 \pm 2.45$ & $28.91 \pm 3.03$ & $41.30 \pm 3.12$ & $38.00 \pm 3.99$ \\
\hline 48 h post & $38.88 \pm 4.01$ & $29.27 \pm 3.86$ & $44.46 \pm 2.50$ & $38.46 \pm 2.09$ \\
\hline
\end{tabular}

CON: Control condition with no occlusion; AOP-50: Experimental condition at 50\% of Arterial Occlusion Pressure; AOP-75: Experimental condition at $75 \%$ of Arterial Occlusion Pressure; AOP-100: Experimental condition at $100 \%$ of Arterial Occlusion Pressure.

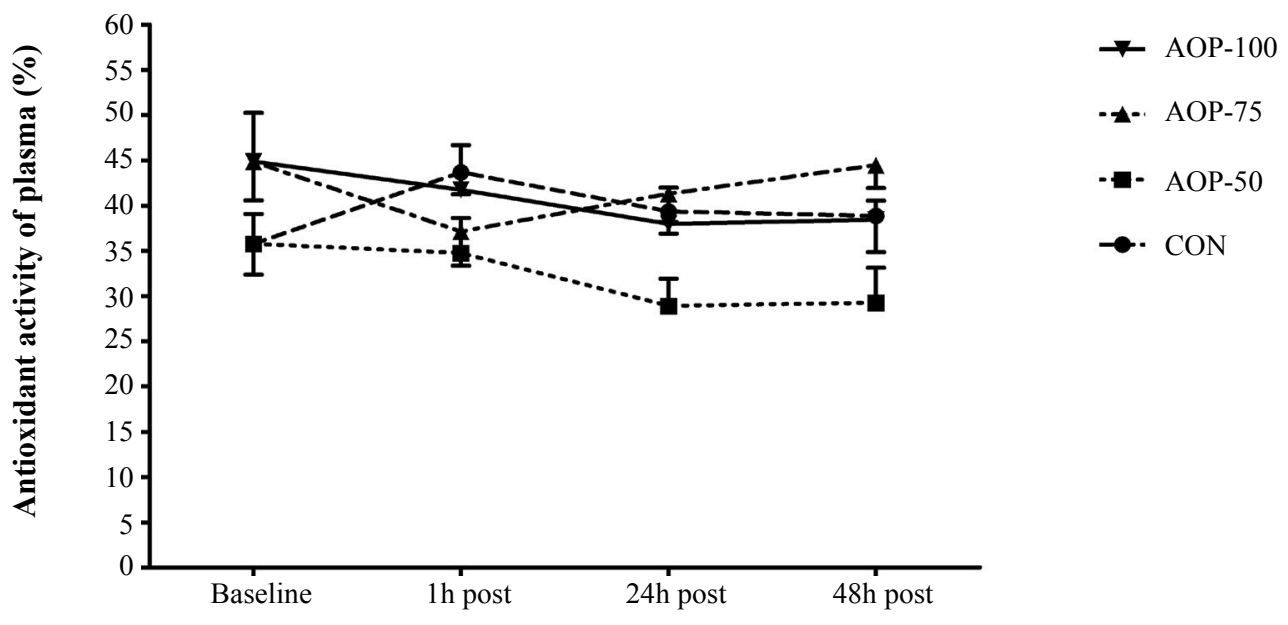

Time points

Figure 2: Comparison of the antioxidant activity of plasma (\%) at rest, $1 \mathrm{~h}, 24 \mathrm{~h}$, and $48 \mathrm{~h}$ after resistance exercise performed at different levels of AOP $(n=12)$. CON: Control condition with no occlusion; AOP-50: Experimental condition at $50 \%$ of Arterial Occlusion Pressure; AOP-75: Experimental condition at 75\% of Arterial Occlusion Pressure; AOP-100: Experimental condition at $100 \%$ of Arterial Occlusion Pressure.

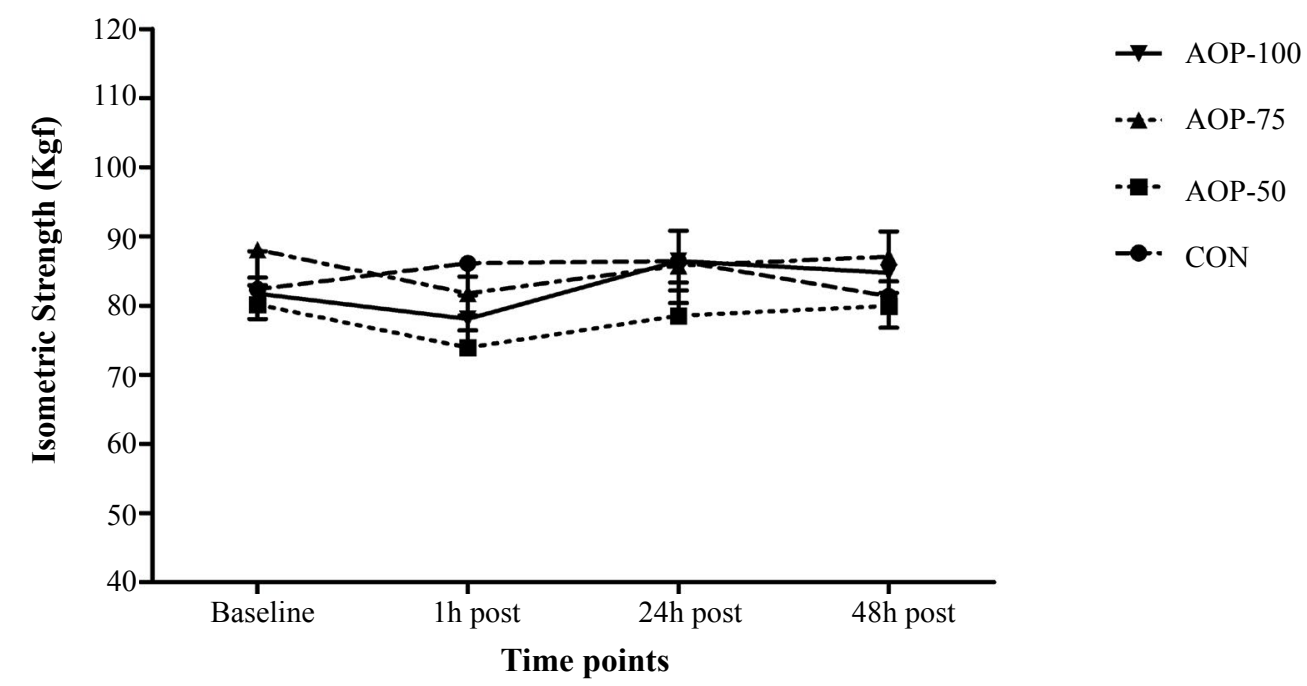

Figure 3: Comparison of isometric strength levels at rest, $1 \mathrm{~h}, 24 \mathrm{~h}$, and $48 \mathrm{~h}$ after resistance exercise performed at different levels of AOP $(n=12)$. CON: Control condition with no occlusion; AOP-50: Experimental condition at $50 \%$ of Arterial Occlusion Pressure; AOP-75: Experimental condition at 75\% of Arterial Occlusion Pressure; AOP-100: Experimental condition at 100\% of Arterial Occlusion Pressure.

Figure 4 presents the serum levels of $\mathrm{CK}$. There was no significant condition $(F=2.63, p=0.07)$ or time $(F=$ $1.25, p=0.31$ ) main effects and no significant condition by time interaction $(F=1.22, p=0.29)$. For plasma levels of LDH (Figure 5), the main effect of condition was not significant $(F=1.10, p=0.12)$ and there was no significant condition by time interaction $(F=0.98, p=0.46)$. However, there was a significant time main effect ( $F$ $=3.29, p=0.048$ ), in which plasma levels of LDH were significant lower $(p<0.01) 24$ h post-exercise $(265.83$ 
Table 3: Comparison of delayed-onset muscle soreness at rest, $1 \mathrm{~h}, 24 \mathrm{~h}$, and $48 \mathrm{~h}$ post-resistance exercise performed at different levels of AOP $(n=12)$.

\begin{tabular}{|c|c|c|c|c|}
\hline \multirow[b]{2}{*}{ Time point } & \multicolumn{4}{|c|}{ AOP pressures } \\
\hline & CON (0\%) & AOP-50 & AOP-75 & AOP-100 \\
\hline Rest & $0.00 \pm 0.00$ & $0.00 \pm 0.13$ & $0.00 \pm 0.22$ & $0.00 \pm 0.17$ \\
\hline $1 \mathrm{~h}$ post & $0.00 \pm 0.11$ & $0.00 \pm 0.41$ & $0.00 \pm 0.56$ & $0.00 \pm 0.42$ \\
\hline $24 \mathrm{~h}$ post & $0.00 \pm 0.25$ & $0.11 \pm 0.19$ & $0.00 \pm 0.29$ & $0.00 \pm 0.37$ \\
\hline $48 \mathrm{~h}$ post & $0.00 \pm 0.11$ & $0.00 \pm 0.08$ & $0.00 \pm 0.08$ & $0.00 \pm 0.33$ \\
\hline
\end{tabular}

CON: Control condition with no occlusion; AOP-50: Experimental condition at 50\% of Arterial Occlusion Pressure; AOP-75: Experimental condition at $75 \%$ of Arterial Occlusion Pressure; AOP-100: Experimental condition at $100 \%$ of Arterial Occlusion Pressure.

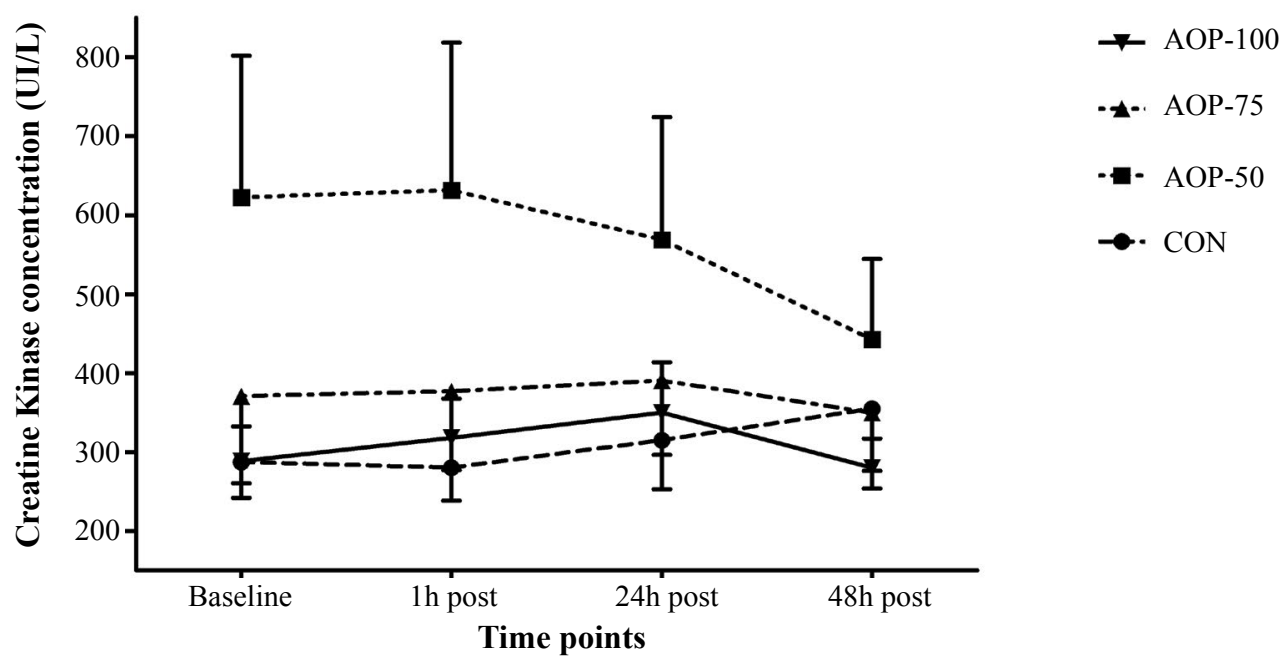

Figure 4: Comparison of creatine kinase concentrations at rest, $1 \mathrm{~h}, 24 \mathrm{~h}$, and $48 \mathrm{~h}$ post-resistance exercise performed at different levels of AOP $(n=12)$. CON: Control condition with no occlusion; AOP-50: Experimental condition at $50 \%$ of Arterial Occlusion Pressure; AOP-75: Experimental condition at 75\% of Arterial Occlusion Pressure; AOP-100: Experimental condition at $100 \%$ of Arterial Occlusion Pressure.
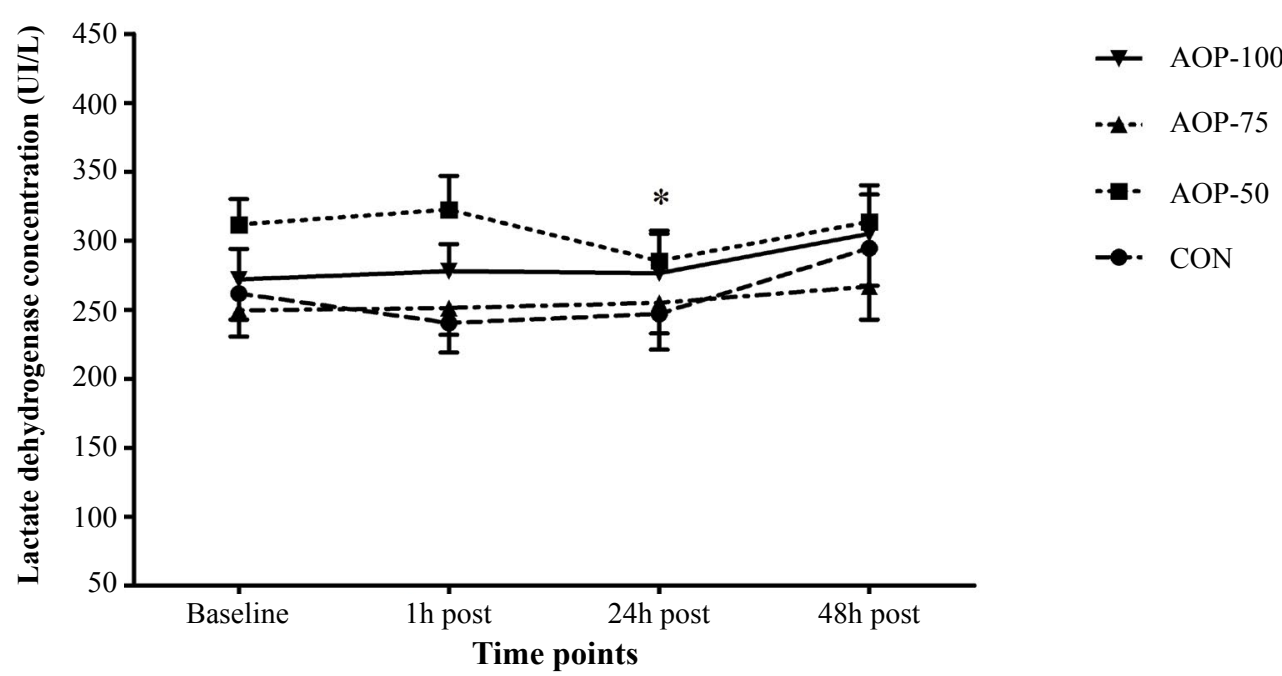

Figure 5: Comparison of the lactate dehydrogenase concentrations at rest, $1 \mathrm{~h}, 24 \mathrm{~h}$, and $48 \mathrm{~h}$ post-resistance exercise performed at different levels of AOP $(n=12)$. CON: Control condition with no occlusion; AOP-50: Experimental condition at $50 \%$ of Arterial Occlusion Pressure; AOP-75: Experimental condition at $75 \%$ of Arterial Occlusion Pressure; AOP-100: Experimental condition at $100 \%$ of Arterial Occlusion Pressure. * Significantly lower than $48 \mathrm{~h}$ post-exercise $(p<0.05)$.

\section{$\pm 17.55 \mathrm{UI} / \mathrm{L})$ compared to $48 \mathrm{~h}$ post-exercise (294.96 \pm $17.51 \mathrm{UI} / \mathrm{L})$.}

There was no significant difference for levels of delayed-onset muscle soreness following any of the post-exercise testing times ( $1 \mathrm{~h}, 24 \mathrm{~h}$ and $48 \mathrm{~h}: \mathrm{p}>0.05$ ) (Table 1 and Table 3).

\section{Discussion}

Even though several studies have investigated BFR exercise-induced oxidative stress and muscle damage $[7,11,23-25]$, this was the first study to analyze oxidative stress and muscle damage responses to resistance exercise performed at different degrees of AOP. The findings 
of the present study do not support our previous hypothesis that applying high levels of AOP would result in higher oxidative stress responses and muscle damage, as well as longer recovery periods.

Our findings are consistent with those from other studies that investigated the relationship between BFR and exercise-induced muscle damage [14,26,27]. Using elastic bands, Wilson, et al. [27] observed a significant elevation in the determinants of hypertrophy without identifying significant elevations in the indicators of muscle damage (muscle soreness, muscle swelling and power) up to $24 \mathrm{~h}$ post-exercise. This supports the idea of using BFR resistance exercise as an effective alternative for promoting significant enhancements in the levels of strength and hypertrophy for those who are unable to train at high loads $[28,29]$. It also supports the relative safety of this method of training [30].

Prolonged reductions in muscle strength have been reported to be one of the most reliable indirect markers of muscle damage [6,31]. In the present study, the maximal isometric strength was measured at $1 \mathrm{~h}, 24 \mathrm{~h}$, and up to $48 \mathrm{~h}$ post-exercise, however, there were no decreases in isometric strength levels at any time point, regardless of the AOP applied. Similar findings were reported by Loenneke, et al. [32] who did not observe any impact of BFR by itself or in combination with exercise (BFR pressure relative to participants' thigh circumference) on levels of isometric strength at $1 \mathrm{~h}$ and $24 \mathrm{~h}$ post-exercise.

Furthermore, there was no increase in the ratings for DOMS for any of the exercise conditions in the current study. Interestingly, different findings were reported by Umbel, et al. [8], who reported increased DOMS ratings above baseline levels at $24 \mathrm{~h}$ and $48 \mathrm{~h}$ post-exercise. However, it is important to note that the authors designed the study so that the experimental group (BFR at $130 \%$ of upper arm SBP applied to the upper thigh) completed the repetitions until muscle failure, however, the control group (not occluded) only completed the same number of repetitions as the experimental group without reaching muscle failure since they were non-occluded.

The serum levels of CK and LDH were also measured as indirect biomarkers of muscle damage. In this regard, Clarkson and Hubal [6] stated that CK levels are expected to rise over $100 \%$ following resistance exercise in comparison to their baseline levels and that it remains elevated for several days following resistance exercise. However, no significant increases from baseline values in the levels of CK and LDH were observed over time for any of the conditions tested. Although LDH levels significantly increased for all conditions $48 \mathrm{~h}$ post-exercise in comparison to $24 \mathrm{~h}$ post-exercise, this difference is likely due to normal daily variations in serum levels of LDH rather than the exercise performed, as no significant dif- ference was observed neither between the conditions tested nor between $1 \mathrm{~h}$ and $48 \mathrm{~h}$ post-exercise. Other studies have shown, that even at high BFR pressures ( $\geq 200 \mathrm{mmHg}$ ), there were no significant changes in the levels of CK at 24 and $48 \mathrm{~h}$ post-exercise [13]. Takarada, et al. [33] applied a BFR pressure of $214 \mathrm{mmHg}$ to young subjects who exercised at $20 \%$ of $1 \mathrm{RM}$ and also did not observe significant elevations in levels of CK at $24 \mathrm{~h}$ post-exercise. Coupled with the findings of the present study, this evidence suggests that BFR exercise does not result in muscle damage, even when the exercise is performed at very high pressures (over $200 \mathrm{mmHg}$ ).

The acute oxidative stress response was also measured in the present study. Oxidative stress is known for its deleterious effects on DNA and skeletal muscle due to the formation of reactive oxygen species, which can also lead to muscle damage $[34,10]$. Since BFR exercise is commonly performed at very low intensities (i.e. $20 \%$ $30 \%$ of $1 \mathrm{RM})$, it is very unlikely that the mechanisms known for evoking muscle damage during traditional resistance training such as physical damage to muscle fibers due to high mechanical stress associated with high intensities and volumes [1,4], is applicable to this model of exercise. Therefore, perhaps a more reasonable explanation for the muscle damage linked to BFR exercise would be more metabolic in nature with increases in hydrogen ions concentrations, stimulation of the endocrine system, or an increase in reactive oxygen species. In this context, previous research has reported that an ischemia-reperfusion protocol has been shown to stimulate the formation of reactive species of oxygen in a rat model [10]. However, no changes in any of the oxidative stress markers (LP and AAP) were observed in the current study. Likewise, Takarada, et al. [33] did not observe alterations in the levels of LP up to $24 \mathrm{~h}$ following BFR exercise. Interestingly, the authors also did not observe any increases in the determinants of muscle damage. Additionally, Garten, et al. [11] observed that BFR combined with low-intensity resistance exercise (30\% of 1RM) actually attenuated the oxidative stress response.

It is known that subsequent bouts of resistance exercise, known as the repeated bout training effect, may provide protective adaptations for muscle and help prevent possible damage [35]. However, it is important to note that the design employed in this study attempted to avoid the repeated-bout effect to tamper the muscle damage response. Even though the participants were submitted to a within individuals cross-over design, there was a large period of recovery time allowed between trials, similar to previous studies [36,37]. Additionally, it seems that protective adaptations from previous bouts of exercise are mainly related to high intensity exercise. In this regard, Nosaka and Newton [38] have shown that submaximal resistance exercise (at $50 \%$ of 1RM) does not exacerbate or offer any protection for additional subsequent bouts of exercise. 
By analyzing collectively, all the variables assessed in the current study, no evidence was found to support the hypothesis that resistance exercise with either partial or total AOP were able to induce muscle damage up to $48 \mathrm{~h}$ post-exercise. Usually, muscle damage is observed following high intensity resistance exercise within 24 to $48 \mathrm{~h}$ post-exercise, and it is generally attributed to the eccentric portions of the contractions performed, in which fewer muscle fibers are recruited in comparison to the concentric contractions. However, Thiebaud, et al. [24] reported that eccentric contractions with BFR did not increase muscle activation or result in prolonged muscle damage up to four days following eccentric exercise. It has also been speculated that muscle damage may occur post BFR exercise due to the formation of reactive oxygen species, from the ischemia/reperfusion response that occurs after releasing blood flow [11]. In this regard, even though total AOP was applied to the subjects of this study, it is still very likely that there was some arterial blood flow into the muscle during the exercise, which might diminish the ischemia/reperfusion process.

It has also been suggested that exercise intensity and exercise volume may play a role in the exercise-induced muscle damage response [1]. Thus, it is worth noting many of the previous BFR studies performed BFR exercise at high volumes (75 reps) [39] or until failure [40]. This is a limitation of the present study, which applied an exercise volume of only 40 reps. It is important to highlight that this moderate exercise volume (4 sets of 10 reps) and it was used in order to standardize all the tested conditions, since the AOP-100 condition would not allow subjects to perform the exercise with a higher number of repetitions. Additionally, the low load traditionally used during BFR resistance exercise $<50 \%$ of 1RM) may be responsible for the absence of muscle damage in this training method. Moreover, we did not measure the oxidative stress and muscle damage responses beyond $48 \mathrm{~h}$ post-exercise; and it may be possible that the measures of damage might occur after 48 $\mathrm{h}$ post-exercise. Finally, this study only included young male resistance trained participants, which makes it difficult to extend these findings to other populations, such as the elderly, women, and the untrained. It is very likely that untrained individuals may have responded differently to resistance exercise performed at different degrees of AOP.

In conclusion, low-intensity resistance exercise with BFR does not result in acute oxidative stress and muscle damage responses in young trained men, regardless of the level of AOP applied during the exercise. From a practical standpoint, BFR resistance training seems to have no detrimental effect on isometric muscle strength and soreness, and a similar recovery time after resistance training at different AOPs could be expected (50, 75 , and $100 \%)$. Moreover, these findings confirm the relative safety of this method of training, however, we encourage future studies to investigate this response in other populations, such as the untrained, women, and the elderly subjects.

\section{Acknowledgments}

The authors would like to thank the participants and the nurses that dedicated their precious time in contribution to this study.

\section{References}

1. Heavens KR, Szivak TK, Hooper DR, Dunn-Lewis C, Comstock BA, et al. (2014) The effects of high intensity short rest resistance exercise on muscle damage markers in men and women. J Strength Cond Res 28: 1041-1049.

2. Nosaka K, Clarkson PM (1994) Effect of eccentric exercise on plasma enzyme activities previously elevated by eccentric exercise. Eur J Appl Physiol Occup Physiol 69: 492-497.

3. Sorichter S, Koller A, Haid C, Wicke K, Judmaier W, et al. (1995) Light concentric exercise and heavy eccentric muscle loading: effects on CK, MRI and markers of inflammation. Int J Sports Med 16: 288-292.

4. Hesselink MK, Kuipers $H$, Geurten $P$, Van Straaten $H$ (1996) Structural muscle damage and muscle strength after incremental number of isometric and forced lengthening contractions. J Muscle Res Cell Motil 17: 335-341.

5. Cutlip RG, Geronilla KB, Baker BA, Chetlin RD, Hover I, et al. (2005) Impact of stretch-shortening cycle rest interval on in vivo muscle performance. Med Sci Sports Exerc 37: $1345-1355$.

6. Clarkson PM, Hubal MJ (2002) Exercise-induced muscle damage in humans. Am J Phys Med Rehabil 81: S52-S69.

7. Loenneke JP, Thiebaud RS, Abe T (2014) Does blood flow restriction result in skeletal muscle damage? A critical review of available evidence. Scand J Med Sci Sports 24: e415-e422.

8. Umbel JD, Hoffman RL, Dearth DJ, Chleboun GS, Manini TM, et al. (2009) Delayed-onset muscle soreness induced by low-load blood flow-restricted exercise. Eur J Appl Physiol 107: $687-695$.

9. Wernbom M, Paulsen G, Nilsen TS, Hisdal J, Raastad T (2012) Contractile function and sarcolemmal permeability after acute low-load resistance exercise with blood flow restriction. Eur J Appl Physiol 112: 2051-2063.

10. Uchiyama S, Tsukamoto H, Yoshimura S, Tamaki T (2006) Relationship between oxidative stress in muscle tissue and weight-lifting-induced muscle damage. Pflügers Arch 452: 109-116.

11. Garten RS, Goldfarb A, Crabb B, Waller J (2015) The Impact of partial vascular occlusion on oxidative stress markers during resistance exercise. Int J Sports Med 36: 542549 .

12. Yasuda $T$, Fukumura $K$, Fukuda $T$, Uchida $Y$, lida $H$, et al. (2014) Muscle size and arterial stiffness after blood flow-restricted low-intensity resistance training in older adults. Scand J Med Sci Sports 24: 799-806.

13. Fujita T, Brechue WF, Kurita K, Sato $Y$, Abe T (2008) Increased muscle volume and strength following six days of low-intensity resistance training with restricted muscle blood flow. Int J KAATSU Train Res 4: 1-8.

14. Loenneke JP, Fahs CA, Rossow LM, Sherk VD, Thiebaud 
RS, et al. (2012) Effects of cuff width on arterial occlusion: implications for blood flow restricted exercise. Eur J Appl Physiol 112: 2903-2912.

15. Loenneke JP, Thiebaud RS, Abe T, Bemben MG (2014) Blood flow restriction pressure recommendations: the hormesis hypothesis. Med Hypotheses 82: 623-626.

16. Laurentino GC, Ugrinowitsch C, Roschel H, Aoki MS, Soares AG, et al. (2012) Strength training with blood flow restriction diminishes myostatin gene expression. Med Sci Sports Exerc 44: 406-412.

17. Evans C, Vance S, Brown M (2010) Short-term resistance training with blood flow restriction enhances microvascular filtration capacity of human calf muscles. J Sports Sci 28 999-1007.

18. Baechle TRT, Earle RWR (2008) Essentials of strength training and conditioning. ( $3^{\text {rd }}$ edn), Human Kinetics.

19. Ohkawa H, Ohishi N, Yagi K (1979) Assay for lipid peroxides in animal tissues by thiobarbituric acid reaction. Anal Biochem 95: 351-358.

20. Brand-Williams W, Cuvelier ME, Berset C (1995) Use of a free radical method to evaluate antioxidant activity. LWT Food Sci Technol 28: 25-30.

21. Nieman DC, Henson DA, Dumke CL, Oley K, McAnulty SR, et al. (2006) Ibuprofen use, endotoxemia, inflammation, and plasma cytokines during ultramarathon competition. Brain Behav Immun 20: 578-584.

22. Beck TW (2013) The importance of a priori sample size estimation in strength and conditioning research. J Strength Cond Res 27: 2323-2337.

23. Karabulut M, Sherk VD, Bemben DA, Bemben MG (2013) Inflammation marker, damage marker and anabolic hormone responses to resistance training with vascular restriction in older males. Clin Physiol Funct Imaging 33: 393-399.

24. Thiebaud RS, Loenneke JP, Fahs CA, Kim D, Ye X, et al. (2014) Muscle damage after low-intensity eccentric contractions with blood flow restriction. Acta Physiol Hung 101: 150157.

25. Thiebaud RS, Yasuda T, Loenneke JP, Abe T (2013) Effects of low-intensity concentric and eccentric exercise combined with blood flow restriction on indices of exercise-induced muscle damage. Interv Med Appl Sci 5: 53-59.

26. Sudo M, Ando S, Poole DC, Kano Y (2015) Blood flow restriction prevents muscle damage but not protein synthesis signaling following eccentric contractions. Physiol Rep 3: e12449.

27. Wilson JM, Lowery RP, Joy JM, Loenneke JP, Naimo MA, et al. (2013) Practical blood flow restriction training increases acute determinants of hypertrophy without increasing indices of muscle damage. J Strength Cond Res 27: 3068-3075.
28. Gualano B, Ugrinowitsch C, Neves M Jr, Lima FR, Pinto AL, et al. (2010) Vascular occlusion training for inclusion body myositis: a novel therapeutic approach. J Vis Exp.

29. Takarada Y, Takazawa H, Ishii N (2000) Applications of vascular occlusion diminish disuse atrophy of knee extensor muscles. Med Sci Sports Exerc 32: 2035-2039.

30. Nakajima $T$, Kurano $M$, lida $H$, Takano $H$, Oonuma $H$, et al. (2006) Use and safety of KAATSU training:Results of a national survey. Int J KAATSU Train Res 2: 5-13.

31. Paulsen G, Mikkelsen UR, Raastad T, Peake JM (2012) Leucocytes, cytokines and satellite cells: what role do they play in muscle damage and regeneration following eccentric exercise? Exerc Immunol Rev 18: 42-97.

32. Loenneke JP, Thiebaud RS, Fahs CA, Rossow LM, Abe $\mathrm{T}$, et al. (2013) Blood flow restriction does not result in prolonged decrements in torque. Eur J Appl Physiol 113: 923931.

33. Takarada $Y$, Nakamura $Y$, Aruga S, Onda T, Miyazaki S, et al. (2000) Rapid increase in plasma growth hormone after low-intensity resistance exercise with vascular occlusion. $J$ Appl Physiol (1985) 88: 61-65.

34. Powers SK, Duarte J, Kavazis AN, Talbert EE (2010) Reactive oxygen species are signalling molecules for skeletal muscle adaptation. Exp Physiol 95: 1-9.

35. McHugh MP (2003) Recent advances in the understanding of the repeated bout effect: the protective effect against muscle damage from a single bout of eccentric exercise. Scand J Med Sci Sports 13: 88-97.

36. Hausswirth C, Louis J, Bieuzen F, Pournot H, Fournier J, et al. (2011) Effects of whole-body cryotherapy vs. far-infrared vs. passive modalities on recovery from exercise-induced muscle damage in highly-trained runners. PLoS One 6: e27749.

37. Pournot H, Bieuzen F, Louis J, Fillard JR, Barbiche E, et al. (2011) Time-Course of changes in inflammatory response after whole-body cryotherapy multi exposures following severe exercise. PLoS One 6: e22748.

38. Nosaka K, Newton M (2002) Repeated eccentric exercise bouts do not exacerbate muscle damage and repair. J Strength Cond Res 16: 117-122.

39. Ozaki H, Yasuda T, Ogasawara R, Sakamaki-Sunaga M, Naito $\mathrm{H}$, et al. (2013) Effects of high-intensity and blood flow-restricted low-intensity resistance training on carotid arterial compliance: role of blood pressure during training sessions. Eur J Appl Physiol 113: 167-174.

40. Patterson SD, Ferguson RA (2010) Increase in calf post-occlusive blood flow and strength following short-term resistance exercise training with blood flow restriction in young women. Eur J Appl Physiol 108: 1025-1033. 